\title{
Rail terminal operations
}

\author{
Nils Boysen ${ }^{1}$ • Dirk Briskorn² ${ }^{2}$ Sigrid Knust ${ }^{3}$
}

Strengthening the railway system is among the top priorities of the long-term transport agendas issued by many national and international authorities. Rail transportation relieves the overloaded road networks and is preferred based on its reduced environmental impact. An important driver to increase the attractiveness of the railway system is to establish more efficient train handling processes in existing railway terminals. Relevant terminals are traditional shunting yards, where railcars are rearranged to trains via a system of switches and shunting tracks, and intermodal terminals, where containers are exchanged between trains and trucks by huge gantry cranes. In all these terminals, multiple stakeholders (e.g., train operators, forwarding companies and terminal operators) and various equipment types (e.g., locomotives, railcars, cranes, containers) have to be coordinated, so that the application of quantitative approaches for planning the operations in rail terminals is highly advisable.

$凶 \quad$ Nils Boysen

nils.boysen@uni-jena.de

http://www.om.uni-jena.de/

Dirk Briskorn

briskorn@wiwi.uni-wuppertal.de

http://www.prodlog.uni-wuppertal.de/

Sigrid Knust

sigrid@informatik.uni-osnabrueck.de

https://www.informatik.uni-osnabrueck.de/knust/

1 Lehrstuhl für Operations Management, Friedrich-Schiller-Universität Jena, Carl-Zeiß-Str. 3, 07743 Jena, Germany

2 Lehrstuhl für Produktion und Logistik, Bergische Universität Wuppertal, Rainer-Gruenter-Str. 21, 42119 Wuppertal, Germany

3 Institut für Informatik, Universität Osnabrück, Wachsbleiche 27, 49090 Osnabrück, Germany 
This special issue is dedicated to operations research methods supporting the planning and scheduling processes in rail terminal operations. From the eleven paper submitted to this special issue, the following three papers have been selected:

Heggen et al. (2018) consider train load planning problems in intermodal terminals and develop a multi-objective model including several practical constraints. The problem is solved exactly by an $\epsilon$-constraint method and heuristically by a multi-directional local search procedure.

Jaehn et al. (2018) study the railcar retrieval problem where railcars having different types must be retrieved in certain quantities from storage tracks such that shunting costs are minimized. After presenting a MIP formulation and showing NP-hardness of the general problem, exact polynomial-time algorithms are proposed for special cases. Furthermore, heuristic solution approaches motivated by current real-world planning routines are analyzed with respect to their worst-case and average performance.

Tschöke and Boysen (2018) address railway terminals in large seaports where freight trains are to be loaded with containers by gantry cranes. Some terminals apply multi-trailer trucks to deliver a long queue of up to a dozen containers toward a rail terminal. The authors consider the problem where to park successive multi-trailer trucks and how to schedule the gantry crane transferring the containers between trucks and trains.

We thank the authors and all reviewers for contributing to this special issue.

Nils Boysen, Dirk Briskorn and Sigrid Knust, February 2018

\section{References}

Heggen H, Braekers K, Caris A (2018) A multi-objective approach for train load planning. OR Spectr. https://doi.org/10.1007/s00291-017-0503-1 (this issue)

Jaehn F, Otto A, Seifried K (2018) Shunting operations at flat yards: retrieving freight railcars from storage tracks. OR Spectr. https://doi.org/10.1007/s00291-017-0495-x (this issue)

Tschöke M, Boysen N (2018) Container supply with multi-trailer trucks: Parking strategies to speed up the gantry crane based loading of freight trains in rail yards. OR Spectr. https://doi.org/10.1007/s00291017-0491-1 (this issue) 